\title{
Bureau d'Etude COMACH : une collaboration Industrie/Ecole pour une synthèse des enseignements en Mécatronique
}

\author{
DUHAYON Eric ${ }^{1}$, ROUCHON Jean-François ${ }^{1}$, LEFEVRE Yvan $^{1}$, HENAUX Carole ${ }^{1}$ et NOGAREDE Bertrand ${ }^{1}$ \\ SAGANSAN Alain ${ }^{2}$, PORTE Guillaume ${ }^{3}$ \\ 1 - Eric.Duhayon@laplace.univ-tlse.fr \\ Groupe de recherche en Electrodynamique (GREM3) \\ INPT-ENSEEIHT LAPLACE 2 Rue Charles Camichel B.P.7122 31071 TOULOUSE Cedex 7
}

2 - Alain.Sagansan@airbus.com

AIRBUS France S.A.S. 316 Route de Bayonne 31060 Toulouse Cedex 03, France

3 - guillaume.jacques.porte@gmail.com

NOVATEM S.A.S. 21 Place Simiot, 33800 BORDEAUX

\begin{abstract}
RESUME : Le bureau d'étude «Conception Machine » $(\mathrm{COMACH})$ proposé aux élèves de $3^{\text {ème }}$ année option TEMA (Transformation de l'énergie et Mécatronique Avancée) du Département Génie Electrique Automatique de l'ENSEEIHT est un enseignement transversal permettant d'effectuer la synthèse des connaissances acquises durant la scolarité. Sur la base d'un cahier des charges de système électromécanique pour l'aéronautique proposé par l'industriel donneur d'ordre (AIRBUS), les élèves, organisés en groupe de projet (4 équipes animées par un élève chef de projet), fournissent une proposition technique complète selon un déroulement d'appel d'offre industriel. Guidés en cela par un industriel expert de ce genre de démarches (NOVATEM), les élèves appliquent les méthodologies académiques enseignées, visualisent leurs limites et appréhendent une approche industrielle pragmatique pour le dimensionnement d'un tel système. Le groupe d'élèves est considéré par Airbus comme un équipementier mis en compétition parmi d'autres. Les élèves se retrouvent donc idéalement placés entre un environnement purement industriel (tendant à favoriser le juste choix technico-économique) et un cadre scolaire de bureau d'études rassurant (favorisant les solutions alternatives et la prise de risques). Les enseignants de la thématique conversion électromécanique du département deviennent une ressource qui donne des conseils et des voies de recherche dans la définition de la solution. En fin de bureau d'étude, les élèves livrent leur solution à Airbus lors d'une présentation devant un jury dirigé par les industriels en justifiant les choix et dimensionnement effectués ainsi que les technologies employées (structure de moteurs, d'électronique, capteurs, système de commande, plans mécaniques et matériaux choisis). Les élèves montrent un réel enthousiasme dans la réalisation de ce projet car ils sont dans une situation qui leur donne une vision réaliste des contraintes du milieu industriel que ce soit d'un point de vue scientifique, technique ou même relationnel et de gestion de projet.
\end{abstract}

Mots clés : Collaboration industrie/école, appel d'offre industriel, système mécatronique, gestion de projet.

\section{INTRODUCTION}

Le présent article traite de la structuration, du déroulement et de la pédagogie associée au bureau d'étude conception machine (BE COMACH). Il expose également une synthèse typique de problématique proposée ainsi que le résultat final fourni par les élèves. Le BE COMACH est le résultat d'un partenariat [1] construit entre AIRBUS, industriel majeur du domaine aéronautique, NOVATEM une petite entreprise MidiPyrénéenne en ingénierie et études techniques et la $3^{\text {ème }}$ année Transformation d'Energie et Mécatronique Avancée (TEMA) du département Génie Electrique et Automatique (GEA) de l'Ecole Nationale Supérieure en Electrotechnique, Electronique, Informatique, Hydraulique et Télécommunications (ENSEEIHT). Cette collaboration conduit à la mise en situation quasi-réelle des élèves du cycle ingénieur sur un appel d'offre pro- posé par AIRBUS afin de concevoir un système mécatronique complet destiné à l'actionnement d'une gouverne de vol de petit aéronef. Les élèves doivent effectuer le travail et s'organiser comme un équipementier afin de fournir une solution pertinente et justifié au cahier des charges exposé. Le groupe d'élèves est conseillé tout au long du BE COMACH par un ingénieur expert de NOVATEM rompu à ce genre d'appels d'offres et par les enseignants de la thématique «conversion électromécanique de l'énergie » du Département GEA. Cette année 2009-2010 constitue la $3^{\text {ème }}$ édition du BE COMACH montrant l'implication forte des acteurs industriels et de l'équipe pédagogique pour que les élèves soient un peu plus proches des réalités et contraintes professionnelles. 


\section{LES INTERVENANTS DU BE COMACH}

\subsection{La promotion de 3ème Année du Département GEA option TEMA (transformation de l'énergie et mécatronique avancée)}

Les élèves de troisième année du cycle d'ingénieur ENSEEIHT de l'option TEMA s'organisent en équipe de projet qui devra travailler pour fournir les attendus d'AIRBUS (Fig. 1). Pour cela, ils devront s'appuyer tant sur les enseignements dispensés au cours de la scolarité ou en cours d'approfondissement lors du premier semestre de 3ème année que sur leur expérience personnelle afin d'adopter la posture permettant l'avancement commun de tout le groupe. En effet, l'évaluation porte sur le résultat final et son degré de validité mais également sur la cohérence globale. Le $\mathrm{BE} \mathrm{COMACH}$ est une occasion unique de mettre en application sur une problématique réaliste toutes les méthodes et outils abordés durant la scolarité.

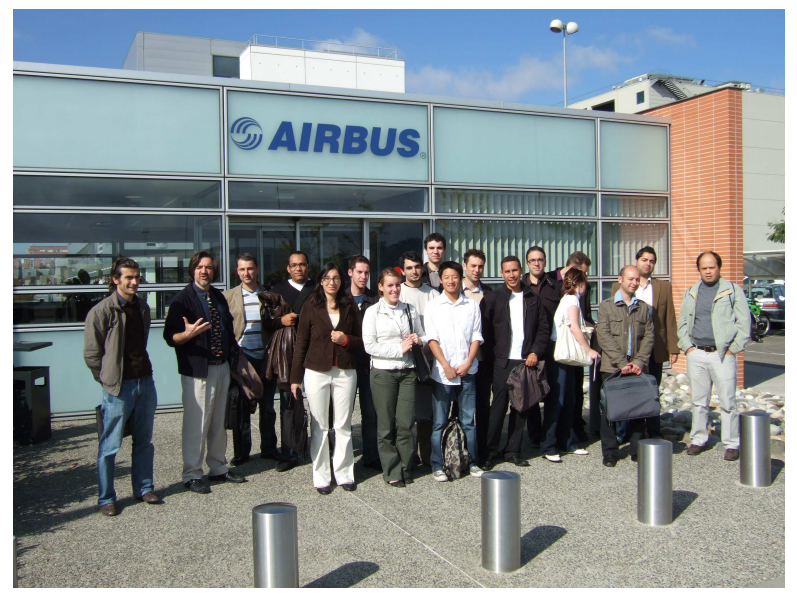

Fig. 1 : La promotion 3GE TEMA 2008-2009 et les encadrants devant l'entrée du site AIRBUS de Saint-Martin du Touch

\subsection{Le partenariat industriel}

La réussite du BE COMACH repose sur l'implication importante dont font preuve les deux industriels. Ces professionnels effectuent avec la promotion TEMA ce qu'ils font tous les jours dans leur activité. Leurs rôles sont différents mais leur intervention permet de placer les élèves dans un cadre plus proche de la réalité que ne pourrait le faire l'institution académique (Fig. 2). Enfin, ces industriels permettent d'effectuer une modification de la relation enseignants/élèves ce qui change fondamentalement l'état d'esprit des étudiants dans l'approche de ce BE.

AIRBUS est le prescripteur de la spécification aéronautique concernant le système mécatronique étudié. Son représentant, Alain SAGANSAN, travaille dans le département commande de vol et plus particulièrement sur les actionneurs électromécaniques. Au cours de la première séance sur le site AIRBUS de Saint Martin du Touch, il présente, au groupe d'élèves, la société, le domaine aéronautique puis focalise le propos sur les commandes de vols électromécaniques. Il est l'interlocuteur privilégié des élèves pour répondre aux questions touchant aux modifications éventuelles du cahier des charges ou au dépassement des caractéristiques maximales assignées (mécanique, bande passante,...). Il a donc le rôle de donneur d'ordres qu'exécutent les élèves.

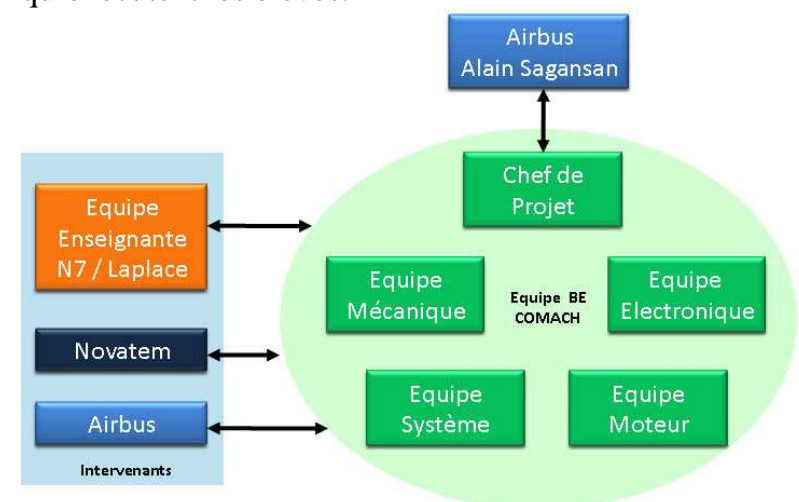

Fig. 2 : Le partenariat Industrie/Ecole/Elèves

NOVATEM est spécialisé dans l'étude de cahiers des charges et dans la réponse innovante et optimisée à des appels d'offres du domaine aéronautique. Plusieurs intervenants de cette société participent au BE COMACH. L'intervenant principal est Guillaume PORTE, ingénieur ENSEEIHT, ayant fait une partie de sa carrière dans la définition et l'étude d'actionneurs électriques pour commandes de vol avant de rejoindre NOVATEM. Ce statut, outre la connaissance des programmes d'enseignements, lui donne un contact privilégié avec les élèves. Il intervient pour transcrire la spécification aéronautique d'AIRBUS en un cahier des charges électromécanique utilisable par le groupe d'élèves. Il traduit et explicite tous les termes et zones floues pour les élèves. Son apport touche également à la mise au point de l'architecture du système étudié et à son pré-dimensionnement par la construction d'un modèle analytique. Ce modèle est basé pour partie sur les enseignements suivis par les élèves qui se rendent donc compte de leur pertinence. L'expert NOVATEM a également une vision globale du système (machine, électronique, commande, contrôle) de la feuille blanche initiale à la réalisation physique ce qui permet d'effectuer un lien entre la vision très théorique des élèves et la mise en œuvre pratique de la solution. Enfin, l'ingénieur NOVATEM complète la formation initiale des élèves car il peut leur expliquer l'étape réalisation et certaines erreurs typiques à éviter pour fiabiliser la structure.

\subsection{L'équipe pédagogique}

Les enseignants-chercheurs de la thématique «conversion électromécanique de l'énergie» du Département GEA, constituant le groupe de recherche en électrodynamique (GREM3) du LAPLACE, interviennent comme des ressources spécialisées afin de fournir aux 
élèves une aide ponctuelle sur des questions préalablement formalisées. Tous les domaines de la thématique pédagogique 3GE TEMA et plus largement des thématiques du Département GEA sont ainsi balayés, de la conversion statique à l'électrodynamique en passant par la mécanique, la commande et les asservissements système.

\section{LE DEROULEMENT DU BE COMACH}

Le BE est cadencé comme un projet industriel autour de quatre rendez-vous principaux (Fig. 3) auxquels participent les industriels :

- Un «kick-Off Meeting (KOM)» dont le but est de présenter les différents intervenants, le contexte aéronautique du projet, les objectifs pédagogiques et la spécification. Au cours de cette réunion réalisée sur le site AIRBUS, les élèves s'auto-organisent en 4 équipes de 4 étudiants et nomment un chef de projet. Les différentes tâches des équipes sont également explicitées,

- La «preliminary design review (PDR) » qui permet de valider que la definition de l'équipement présenté par les élèves soit en conformité avec la spécification.

- La «Detailed Design Review (DDR)» permettant de valider la définition détaillée de l'équipement vis-à-vis de la spécification,

- La «Final Review » constitue le rendez vous final de présentation de la solution technologique optimisée devant un jury mixte présidé par les industriels.

Jusqu'à la PDR, les équipes travaillent en commun afin d'obtenir le pré-dimensionnement au premier ordre de l'architecture du système envisagé. Une fois validée, cette structure est affinée par le travail individuel des équipes spécialisées dans les 4 domaines suivants : Machine, Electronique, Mécanique et système. Le rôle du chef de projet est de favoriser les échanges d'informations entre les équipes, de détecter et résoudre les difficultés techniques, organisationnelles et éventuellement humaines et enfin d'assurer le contact avec le donneur d'ordre industriel.

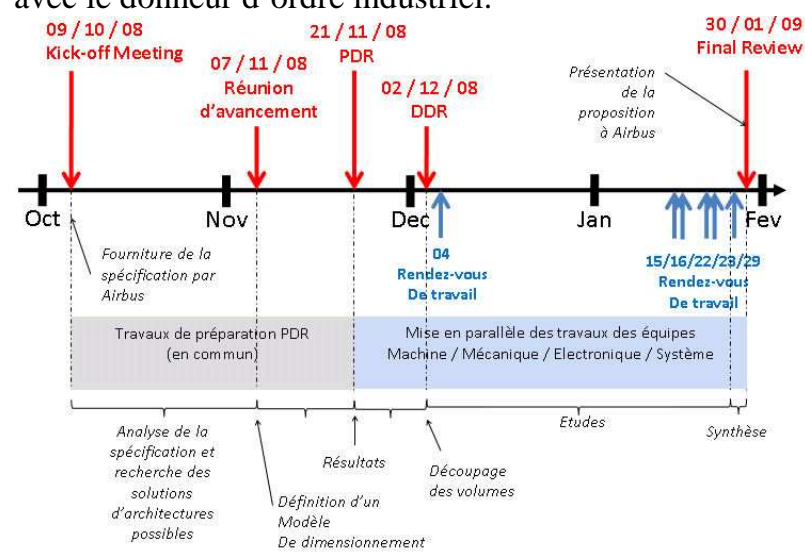

Fig. 3 : Planification type du BE COMACH

L'ensemble des points de rencontre (4x4 hTD) exposés ci-dessus s'intègrent dans le volume d'heures allouées (36 hTD) du programme pédagogique de l'option de $3^{\text {ème }}$ année TEMA. Les créneaux restants permettent aux élèves d'accéder aux moyens informatiques et de solliciter sur des points précis le conseil des enseignants. La réussite du BE COMACH réside également dans l'implication des élèves au travers des réunions et travaux personnels effectués en dehors des créneaux définis. Ces rencontres de travail sont organisées par le chef de projet avec une réponse positive de la part de toute la promotion ce qui montre leur enthousiasme. Ce travail personnel conséquent, évalué au minimum à $40 \mathrm{~h}$, entraine principalement une réduction du temps alloué à leur recherche de stage pour leur projet de fin d'études. De plus, sous l'impulsion des élèves, le traitement de problèmes spécifiques rencontrés en $\mathrm{BE}$ $\mathrm{COMACH}$ sont abordés lors d'autres enseignements proches thématiquement (BE mécanique, TER vibrations, simulation électromagnétique) stimulant des échanges entre enseignants et étudiants, ressentis comme très positifs, sur un système bien appréhendé par les élèves ce qui renforce le caractère transversal du BE COMACH.

\section{LE CAHIER DES CHARGES TYPE}

\subsection{Contrôle des surfaces de commande de vol}

Nous développons les points principaux du cahier des charges fourni à la promotion 3GE TEMA 2008-2009.

Le cahier des charges donne les principales exigences et caractéristiques pour l'étude d'un actionneur électromécanique destiné à contrôler les gouvernes d'un drone (Fig. 4).

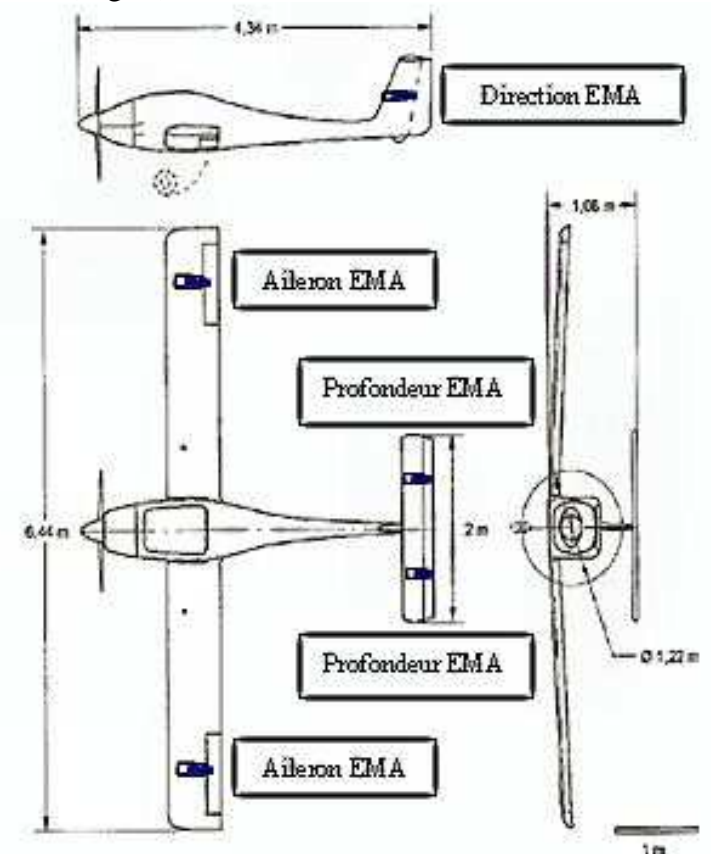

Fig. 4 : Vue du drone d'étude du BE COMACH. 


\subsection{Détail des exigences demandées à l'actionneur}

Les spécifications principales utilisées par les élèves dans le dimensionnement au premier ordre de l'actionneur sont maintenant exposées.

\subsubsection{Description générale}

L'équipement est une boite étanche qui comprend un moteur électrique à courant continu sans balais avec son boitier de gestion électronique, un dispositif de mesure et de limitation d'effort et un capteur de position de la tige de sortie.

\subsubsection{Fonctions assurées par l'actionneur}

L'actionneur doit pouvoir être monté sur n'importe quelle gouverne et assurer un mode actif d'actionnement (positionner la gouverne avec les efforts et les vitesses spécifiées, fournir la position de la sortie mécanique et asservir la gouverne en position) et un mode amorti en cas de perte de puissance électrique (génération d'un effort résistant dépendant linéairement de la vitesse). L'actionneur doit être capable de fournir un effort maximal et continu de $300 \mathrm{~N}$ quelque soit la position gouverne pour une course fonctionnelle de $40 \mathrm{~mm}$ (42mm maximum de butée à butée). En outre, l'équipement complet ne devra pas dépasser une masse de $0.50 \mathrm{~kg}$ (Fig. 5).

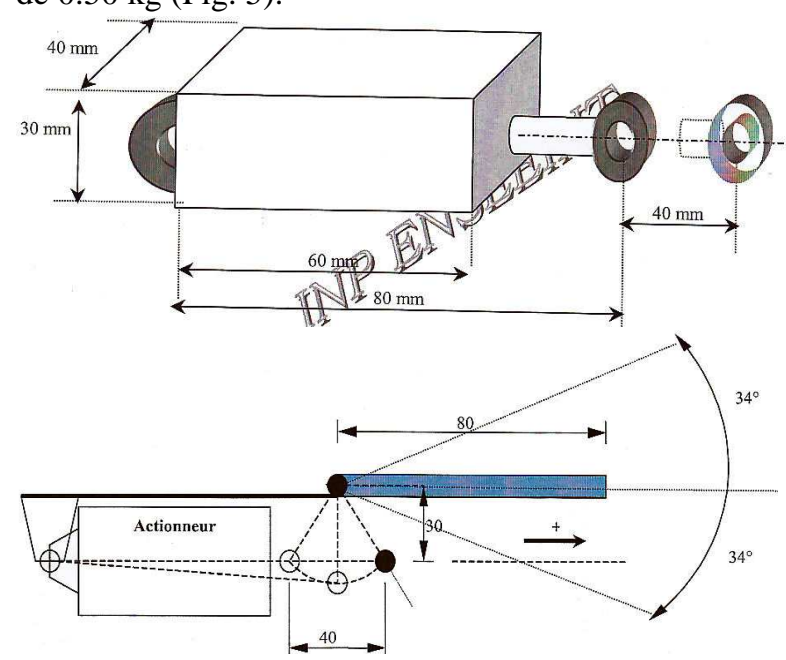

Fig. 5 : Vue schématique de l'actionneur linéaire et de la cinématique gouverne.

\subsubsection{Spécifications du dispositif}

La vitesse de l'équipement sera supérieure ou égale à $40 \mathrm{~mm} / \mathrm{s}$ quelque soit l'effort délivré et sa consommation électrique ne devra pas dépasser $100 \mathrm{~W}$ prélevée sur un bus continu de $48 \mathrm{~V}$ (40 à 50 VDC). La température de peau de l'actionneur ne dépassera pas $110^{\circ} \mathrm{C}$ en considérant une température ambiante de $60^{\circ} \mathrm{C}$ et une convection naturelle. Le dispositif comprend également un capteur de déplacement dont l'alimentation, assurée par le calculateur, est alternative $7 \mathrm{~V}-2 \mathrm{kHz}$.

\section{LA SOLUTION PRECONISEE A LA FINAL REVIEW DU BE COMACH}

Dans un premier temps, nous exposons de manière synthétique le travail réalisé par chaque équipe projet. Les illustrations présentes dans cette partie sont issues du travail fourni. Les élèves utilisent les outils logiciels mis à leur disposition dans l'espace numérique du département GEA. Outre, les éléments classiques des suites bureautiques couramment employés, ils utilisent principalement Matlab® et BondGraph ${ }^{\circledR}$ pour les simulations système, PSIM® pour la simulation de la partie électronique et ANSYS ${ }^{\circledR}$ pour la modélisation multi-physique par éléments finis.

\subsection{Equipe Machine}

Cette équipe doit réaliser pour la PDR un panorama des structures opérationnelles des machines envisageables pour répondre aux exigences. Une hiérarchisation est effectuée afin de mettre en avant la structure la plus appropriée. Sur la base des équations analytiques théoriques exposées dans les cours suivis à l'ENSEEIHT [2], les élèves réalisent un prédimensionnenent de cette solution à partir des données couple-vitesse (au niveau moteur) tirées de la spécification par l'équipe mécanique (à partir de la vitesse de braquage souhaitée et de l'effort maximal fourni). L'approche analytique permet d'obtenir avant la PDR la faisabilité d'un actionneur répondant aux exigences dans le volume donné. Ce prédimensionnement validé en PDR est ensuite finalisé par une étude plus précise des divers paramètres machine (nombre de spires, valeur des inductances et des dimensions des aimants et des encoches). Cette finalisation inclut une étude numérique pour valider les niveaux de saturation magnétique des culasses et des formes d'onde des forces électromotrices et du couple (Fig. 6).

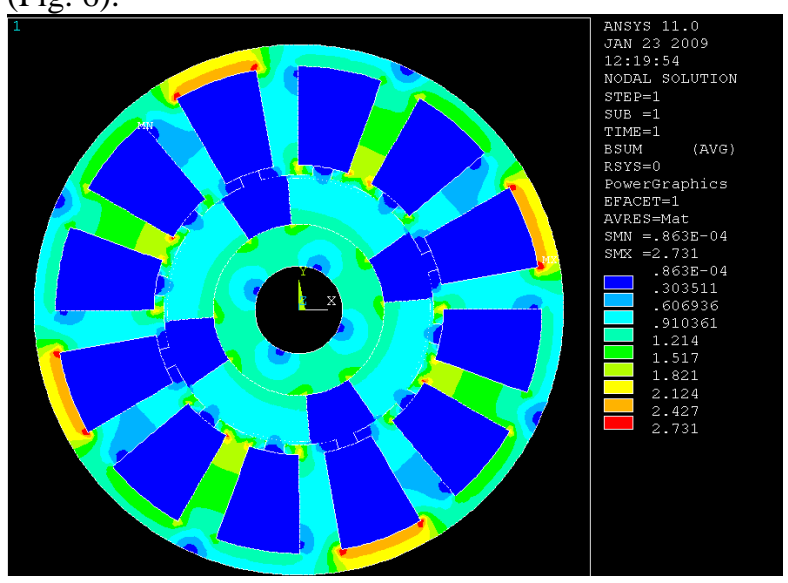

Fig. 6 : Cartographie par éléments finis de l'induction dans l'actionneur

\subsection{Equipe Electronique}

Cette équipe doit choisir la stratégie de commande du moteur et effectuer le design de la carte électronique avec le choix et l'implantation des composants (ségré- 
gation puissance, commande). Elle est également chargée de la simulation des différentes phases de fonctionnement de l'équipement (modes actif et amorti) avec l'évaluation de l'impact thermique.

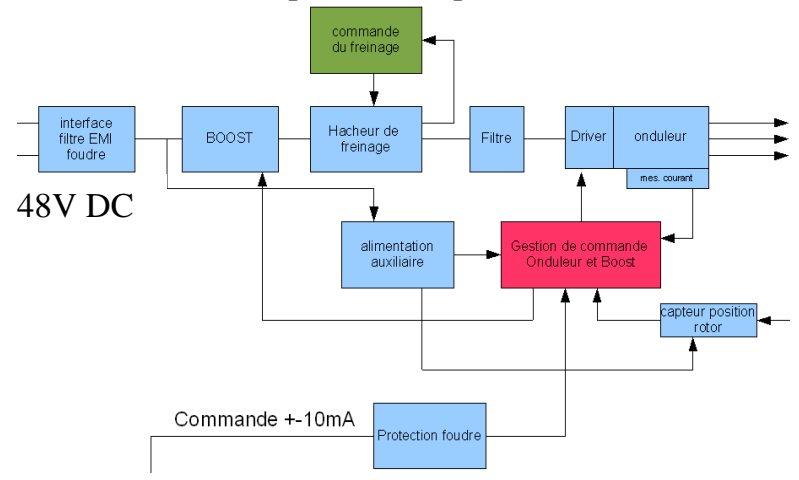

Fig. 7 : Synoptique de l'électronique de commande

La structure du convertisseur d'alimentation dépend fortement du choix de la machine mais reste classique (Fig. 7) et la difficulté majeure réside dans le choix d'un composant industriel intégrable dans la boîte. Une fois la PDR passée, les élèves simulent les signaux d'alimentation machine à partir des informations issues du capteur de position et du signal de commande (Fig. 8) afin de déplacer correctement la gouverne mais également à partir des capteurs internes à la machine (effet Hall) afin d'assurer l'asservissement de celle-ci.

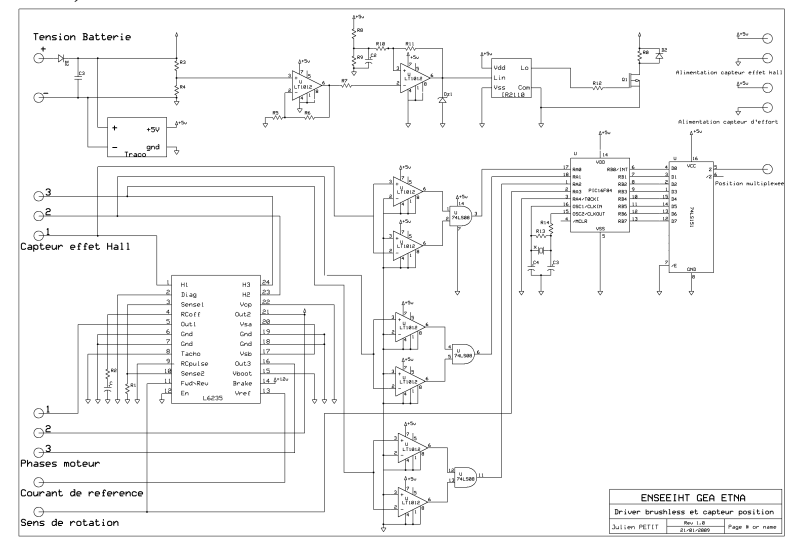

Fig. 8 : Schéma électronique de la carte complète

\subsection{Equipe Système}

Cette équipe doit analyser le système dans sa globalité d'un point de vue des contraintes et performances liées à l'architecture choisie et à son environnement. Elle effectue également la modélisation du système (Fig. 9) afin de qualifier sa plage de réponse dynamique.

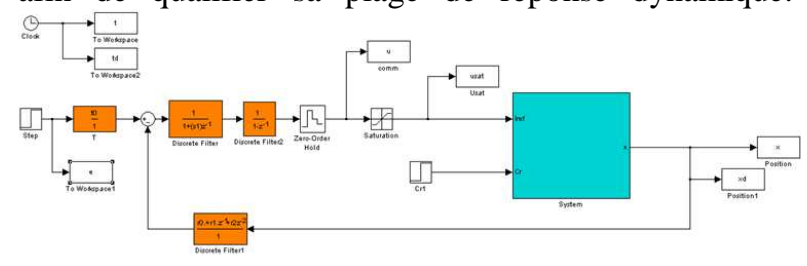

Fig. 9 : Schéma bloc typique implémenté par les élèves.
Les élèves doivent déterminer les régulateurs nécessaires au contrôle et à l'asservissement de l'équipement et valider les plages de fonctionnement en fréquence et en amplitude décrites dans le cahier des charges (Fig. 10).

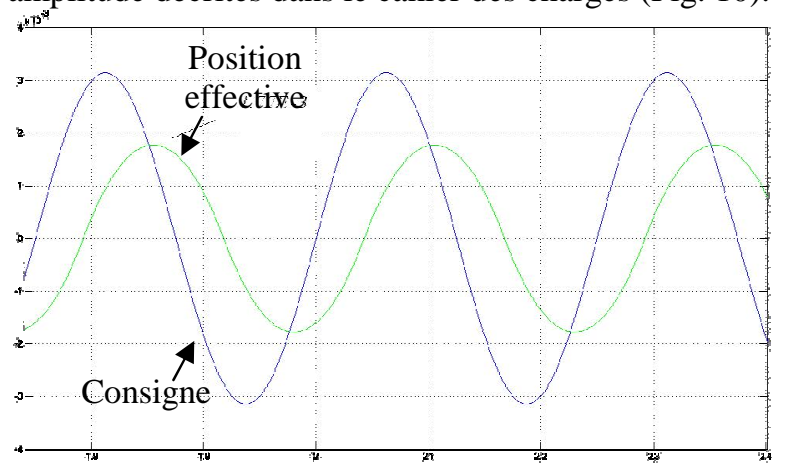

Fig. 10 : Réponse du système à une commande sinusoüdale en fréquence ( $4 \mathrm{~Hz})$ d'amplitude maximale ( $40 \mathrm{~mm}$ de course).

\subsection{Equipe Mécanique}

L'équipe Mécanique est chargée du choix des matériaux et du calcul des éléments de réduction de vitesse et de limitation du couple. Mais plus globalement, elle effectue le calcul des raideurs et jeux de fonctionnement et l'intégration finale des différents constituants dans le volume assigné. Elle crée une vue 3D sous INVENTOR® (Fig. 11) permettant d'évaluer les masses, les points de fixation et édite une liasse de plan sommaire.

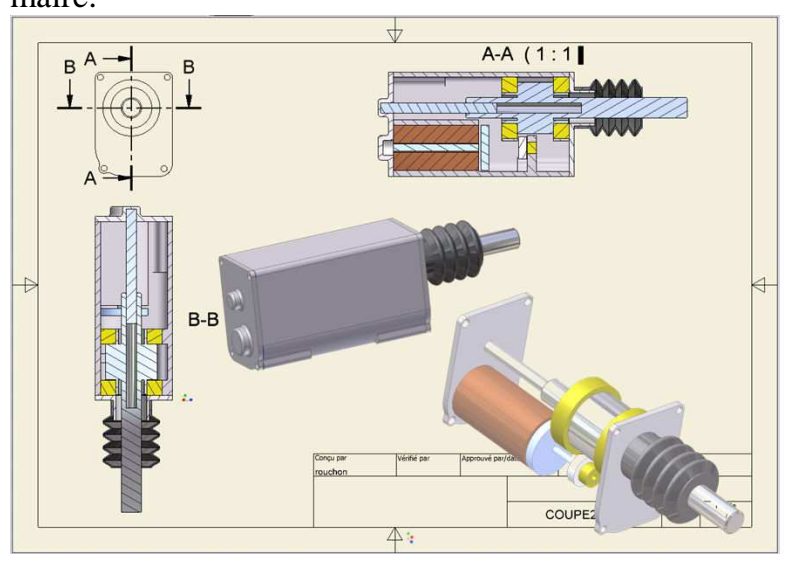

Fig. 11 : Vue en coupe et $3 D$ de l'implantation des éléments mécaniques de la solution proposée.

\subsection{Liaisons entre équipes}

Chaque équipe traite principalement d'un aspect du projet ce qui pourrait nuire à la vision transversale du système électromécanique conçu. Cependant, les échanges fournis entre équipes sous l'impulsion du chef de projet, des jalons fixés ainsi que la nécessaire interdépendance des équipes sur le plan technologique renforcent la coopération des équipes et élargissent le spectre de connaissances de chacune des équipes. 


\section{INTERET PEDAGOGIQUE DU BE COMACH}

\subsection{Le BE COMACH dans le programme pédago- gique de l'option de 3ème année TEMA}

Le caractère transversal du BE COMACH se justifie pleinement dans la formation vis-à-vis de son arrivée en dernière année du cycle d'ingénieur. En effet, il apparaît indispensable de réaliser une synthèse des enseignements (approches théoriques, outils et méthodes associées) dispensés en $1^{\text {ère }}, 2^{\text {ème }}$ et $3^{\text {ème }}$ année du Département GEA [3]. La synthèse effectuée s'avère d'autant plus évidente que l'ossature du BE COMACH repose sur les unités d'enseignements dispensées en $3^{\text {ème }}$ année TEMA (Fig. 12).

$\begin{aligned} & \text { Physique des dispositifs } \\ & \text { électromagnétiques } \\ & \text { (Électrodynamique, Modélisation phenomenenes couplés } \\ & \text {.Couplage électromécanique milieux fluides) }\end{aligned}$
$\begin{aligned} & \text { Conversion électromécanique } \\ & \text { de l'énergie } \\ & \text { (Conception des machines et actionneurs électromécaniques, } \\ & \text { Conception mécanique, Optimisation) }\end{aligned}$
$\begin{aligned} & \text { Architecture des } \\ & \text { systèmes mécatroniques } 42 \mathrm{HC}, 8 \mathrm{BE}, 40 \mathrm{TER} \\ & \text { (Conversion statique, Actionneurs mécatroniques, CEM) }\end{aligned}$
$\begin{aligned} & \text { Contrôle, Cde et diagnostics } \\ & \text { des syst. Mécatroniques } 68 \mathrm{HC}, 16 \mathrm{BE}, 40 \mathrm{TER} \\ & \text { (Modélisation, Identification, diagnostics, Stratégies de } \\ & \text { commandes, Estimation fitrage) }\end{aligned}$

Fig. 12 : Structure des enseignements dispensés en $3^{\text {ème }}$ année TEMA.

Débordant du cadre des spécialités dispensées au Département GEA, le BE COMACH se révèle également un bon exercice d'application des enseignements de gestion de projet suivis par les élèves dont ils mettent en œuvre certains outils (diagramme de Gantt, chemin critique) et qu'ils complètent par l'approche professionnelle que leur montrent les industriels (cotation, chiffrage, heures passées). Enfin, un couplage est effectué avec le service de langues qui mène à la réalisation d'une présentation orale préliminaire en langue anglaise, couramment employée dans le monde industriel et notamment chez AIRBUS.

\subsection{Les élèves deviennent « Acteurs »}

Le BE COMACH est l'occasion donnée aux élèves de prendre en charge un projet concret du stade d'analyse d'un cahier des charges à la mise en plan préalable à la réalisation pratique. Aussi, ils nomment un chef de projet qui doit favoriser les échanges constructifs d'informations entre les équipes. De plus, il doit gérer les interventions des enseignants (pris dans le sens de conseil) en fonction de leurs contraintes. La relation enseignants/élèves se trouve positivement modifiée car l'intervenant traite directement des problèmes soulevés par les élèves ce qui nécessite une formulation adéquate d'une problématique et favorise l'acquisition de connaissances. En effet, l'enseignant peut donc expliquer très concrètement et résoudre la difficulté ou bien rediriger les élèves en donnant des voies de recherches ce qui favorise leur apprentissage des méthodes de résolution.
En ce sens, l'objectif du BE COMACH qui est à l'origine de faire jouer un rôle aux élèves est atteint et donne depuis maintenant trois années de fonctionnement pleine satisfaction tant aux élèves qu'aux enseignants. Cependant, cette réussite nécessite une contre partie en ce qui concerne le travail supplémentaire des élèves, qui par l'enthousiasme dont ils font preuve, s'avère élevé (évaluée à $40 \mathrm{~h}$ de travail personnel).

\subsection{L'évaluation du BE COMACH}

La note finale donnée aux élèves est basée sur une évaluation des «délivrables » rendus à AIRBUS et commentés aux industriels lors de la «Final Review » dans le cadre d'une présentation orale. Si un écart flagrant est constaté dans l'implication de chacune des équipes, la note globale, qui se veut l'image du travail en équipe, peut se voir différenciée. Cette note constitue l'équivalent de 3 crédits ECTS.

\section{CONCLUSION}

Mis en place au sein de la formation d'Ingénieur du Département de formation Génie Electrique et Automatique de l'ENSEEIHT depuis maintenant 3 années, le $\mathrm{BE} \mathrm{COMACH}$ constitue un très bon exemple de mise en œuvre d'une pédagogie par projet spécifique, car préalablement basée sur une acquisition antérieure des connaissances fondamentales. Aussi, le BE COMACH, programmé en fin de cursus, permet de réaliser une synthèse parfaite des différents domaines abordés au cours des 4 semestres précédents et réalise ainsi le lien entre les matières dispensées au Département GEA.

Le partenariat industriel à la base de la création du $\mathrm{BE}$ $\mathrm{COMACH}$, associé à une redistribution des rôles qui place l'enseignant comme une ressource et l'industriel comme donneur d'ordre constitue, de notre point de vue, l'apport original de cette forme pédagogique.

Soulignons aussi que cette forme d'enseignement renforce la perception qu'ont les élèves du métier d'ingénieur concepteur, difficile à appréhender dans un cadre académique plus classique [4].

\section{Bibliographie}

[1] A.P. Massey,. V. Ramesh,. V. Khatri, "Design, development, and assessment of mobile applications: the case for problem-based learning", IEEE Transactions on Education,Vol. 49, Issue: 2, pp. 183 - 192, 2006.

[2] B. Nogarede, D.ominique Harribey, Yvan Lefèvre, ançois Pigache, " Platine d'expérimentation MicroMAG : A la découverte de la machine synchrone autopilotée ", Cetsis 2007, Bordeaux, France, 2007.

[3] B. Nogarede, "Electrodynamique appliquée Bases et principes physiques de l'électrotechnique", Sciences sup, Editions DUNOD, ISBN 210 007314, 2005.

[4] C. A. Land, "The Future Engineers and Scientists: You Need Them? I Have Them. Lots of Them", OCEANS 2008 MTS/IEEE conference proceedings, pp. 1 - 7, Québec, 2008. 